\title{
VALOR PREDICTIVO DE LA ESTIMULACIÓN ELÉCTRICA DE LOS TORNILLOS PEDICULARES TORÁCICOS EN LA MAL POSICIÓN MEDIAL DE LOS MISMOS EN LA INSTRUMENTACIÓN DE CIRUGÍA DE COLUMNA
}

\author{
VALOR PREDITIVO DA ESTIMULAÇÃO ELÉTRICA EM CASOS DE MAL POSICIONAMENTO \\ DE PARAFUSOS PEDICULARES TORÁCICOS NA CIRURGIA DE COLUNA
}

\author{
PREDICTIVE VALUE OF ELECTRICAL STIMULATION IN CASES OF MALPOSITION \\ OF THORACIC PEDICLE SCREWS IN SPINE SURGERY
}

Pedro Cortés García ${ }^{1}$, Mario Herrera Pérez ${ }^{2}$, Pedro Pérez Lorensu4, Bernabé Déniz Rodríguez ${ }^{2}$, Patricia Sánchez Hernández ${ }^{3}$, Ángeles Ayala Rodrigo ${ }^{3}$

RESUMEN

Objetivo: Demostrar si la técnica de estimulación eléctrica permite la detección de la mal posición medial de los tornillos pediculares torácicos. Resultados: Se analizaron 421 tornillos torácicos. Tuvimos alertas a la estimulación en 25 (5,93\%) de los casos. A todos los pacientes se les realizó radiografía posoperatoria demostrando 22 tornillos (5,2\%) medializados. Realizamos TAC en 17 pacientes (37\%), con ningún tornillo en posición 1 y 10 tornillos en posición 2 (8,5\%). Se consideraron tornillos medializados los que tenían respuesta positiva a estimulación inferior a $6 \mathrm{~mA}$. Conclusiones: la estimulación eléctrica nos ha permitido reducir el riesgo de posición medial de los tornillos torácicos, minimizando además el uso de radiografía intraoperatoria.

Descriptores: Tornillos pediculares; Instrumentación vertebral; Monitorización neurofisiológica intraoperatoria.

\section{RESUMO}

Objetivo: Demonstrar se a técnica de estimulação elétrica permite a detecção de mal posicionamento medial dos parafusos pediculares torácicos. Resultados: Foram analisados 421 parafusos torácicos. Os alertas da estimulação ocorreram em 25 (5,93\%) dos casos. Todos os pacientes foram submetidos à radiografia pós-operatória que demonstrou 22 parafusos (5,2\%) medializados. Realizamos TC axial em 17 pacientes (37\%), e não se encontrou nenhum parafuso na posição 1, sendo que 10 parafusos estavam na posição 2 (8,5\%). Foram considerados parafusos medializados os que apresentaram resposta positiva à estimulação inferior a 6 mA. Conclusões: a estimulação elétrica permitiu a redução de risco de posição medial dos parafusos torácicos, minimizando o uso de radiografias intraoperatórias.

Descritores: Parafusos pediculares; Instrumentação vertebral; Monitoração neurofisiológica intraoperatória.

\section{ABSTRACT}

Objective: To demonstrate that the electrical stimulation technique allows the detection of medial malpositioning of thoracic pedicle screws. Results: We analyzed 421 thoracic screws. Stimulation alerts occurred in 25 (5.93\%) cases. All patients underwent postoperative radiographs showing 22 medialized screws (5.2\%). Axial CT scans were performed on 17 patients (37\%), and no screw was found in position 1 and 10 of them were in position 2 (8.5\%). Medialized screws were considered those who had a positive response to stimulation of less than 6 mA. Conclusions: Electrical stimulation allowed a reduction of risk of medial positioning of thoracic screws, minimizing the use of intraoperative radiographs.

Keywords: Pedicle screws; Spinal instrumentation; Intraoperative neurophysiological monitoring.

\section{INTRODUCCIÓN}

La instrumentación vertebral mediante el uso de tornillos pediculares ha ganado popularidad respecto al tradicional uso de ganchos o sistemas híbridos, debido fundamentalmente a tres características: poseer mayor capacidad de corrección tridimensional de la deformidad raquídea, mayores índices de fusión de las artrodesis, así como menores índices de revisión ${ }^{1}$. Pero, a pesar de su uso generalizado, la instrumentación transpedicular no está exenta de riesgos, y especialmente en la región torácica, supone un verdadero reto para el cirujano de raquis 1,3 .

Por otra parte, la peculiaridad del pedículo de la vértebra torácica es única ${ }^{2}$, siendo más estrechos que a nivel lumbar, con mayores cambios de orientación en caso de deformidades, y con malformaciones congénitas en muchos casos, como la ausencia de canalización de los mismos. Si a esto añadimos la cercanía de estructuras vitales en esta región como son la médula espinal y los grandes vasos, nos encontramos con un recurso quirúrgico de alta complejidad técnica y cuyas complicaciones son potencialmente peligrosas.

Como en la columna lumbosacra, la malposición inferior, superior o lateral de los tornillos torácicos puede lesionar raíces nerviosas, sin embargo, es la malposición medial de los mismos la más preocupante, pues la localización intracanal de dicho tornillo puede poner en peligro a la propia médula espinal, amenazando la integridad neurológica de nuestros pacientes.

1. Jefe de Sección de Columna Vertebral - Complejo Hospital Universitario de Canarias. La Laguna, Tenerife (Islas Canarias), España.

2. Médico Adjunto, Servicio de Cirugía Ortopédica y Traumatología B. Complejo Hospital Universitario de Canarias. La Laguna,Tenerife (Islas Canarias), España.

3. Médico Residente, Servicio de Cirugía Ortopédica y Traumatología - Complejo Hospital Universitario de Canarias. La Laguna, Tenerife (Islas Canarias), España.

4. Médico Adjunto, Servicio de Neurofisiología - Complejo Hospital Universitario de Canarias. La Laguna, Tenerife (Islas Canarias), España.

Trabajo realizado na Unidad de Columna Vertebral. Servicio de Cirugía Ortopédica y Traumatología B. Complejo Hospital Universitario de Canarias. La Laguna, Tenerife (Islas Canarias), España. Correspondencia: Hospital Universitario de Canarias carretera La cuesta-taco sin numero. La Laguna. Santa Cruz deTenerife. España. E-mail: pedro.cortes49@gmail.com 
En este sentido, y para detectar la malposición de los tornillos pediculares torácicos, se han diseñado diversas técnicas que comprenden la comprobación manual por palpador, la radioscopia intraoperatoria, la laminotomía y los estudios de conducción eléctrica ${ }^{3}$. Los estudios de conducción eléctrica están basados en la premisa de la resistencia eléctrica dentro del pedículo intacto ${ }^{2}$. Su eficacia ha sido bien documentada en pedículos lumbares ${ }^{7}$, mientras que la literatura muestra resultados variables en la columna torácica ${ }^{4,5}$ Actualmente, es posible evaluar el orificio del tornillo dentro del pedículo electrofisiológicamente, existiendo guías publicadas de umbrales de estimulación para la cirugía de columna lumbosacra ${ }^{4,5}$. En el año 2003, Shi y cols. describieron el uso de guías similares para la instrumentación torácica ${ }^{5}$, recomendando usar la musculatura intercostal para monitorizar las vértebras torácicas superiores.

En base a estos hallazgos el objetivo de nuestro estudio es demostrar si la técnica de estimulación eléctrica de los tornillos pediculares torácicos permite la detección de la malposición medial de los mismos durante el proceso quirúrgico de instrumentación espinal.

\section{MATERIAL Y MÉTODOS}

Se realizó un estudio prospectivo con un total de 46 pacientes intervenidos de cirugía de raquis que requiriese instrumentación y fusión vertebral en el período 2007-2011.

El protocolo anestésico consistió en una anestesia general total intravenosa (TIVA) con propofol $(6-7 \mathrm{mg} / \mathrm{kg} / \mathrm{h})$ y remifentanilo (0.1-0.5 mg/Kg/min). Se utilizó como relajante muscular rocuronio (0.6-1 mg/kg) en dosis única de inducción, sin bloqueo residual en el momento de la instrumentación.

Se utiliza un equipo de monitorización neurofisiológica intraoperatoria (Cadwel| ${ }^{\circledR}$ Cascade Elite de 32 canales), agujas subdérmicas monopolares pareadas $12 \times 0.40 \mathrm{~mm}$ para recoger las respuestas musculares (Neuroline Twisted Pair Subdermal Ambu ${ }^{\circledR}$ ), agujas en forma de sacacorchos para la estimulación eléctrica transcraneal y recepción cortical de los PESS (Subdermal Corkscrew Ambu ${ }^{\circledR}$ ) y electrodos de superficie (Neuroline 72015-K Ambu ${ }^{\circledR}$ ) para la estimulación nerviosa periférica. El electrodo de estimulación monopolar para tornillo pedicular es el Disposable Pedicle Screw Probe de 3 mm (Magstim ${ }^{\circledR}$ ).

Los potenciales evocados motores mediante estimulación eléctrica transcraneal se realizan estimulando con trenes de 7 impulsos de $50 \mu \mathrm{s}$ de duración y con un intervalo inter - estímulo de 4 ms. La recepción muscular se obtiene desde los músculos APB y AH bilateralmente.

Los potenciales evocados somatosensoriales se obtienen estimulando eléctricamente ambos nervios tibiales posteriores con electrodos de superficie y recogiendo las respuestas periféricas en hueco poplíteo y las corticales a nivel Cz-Fz.

La estimulación de tornillos pediculares se realiza mediante el electrodo monopolar y un tren de estímulos de 4 segundos de duración de hasta $10 \mathrm{~mA}$ de intensidad. Los electrodos de registro entre los niveles $T_{2}-T_{6}$ se colocan en la musculatura intercostal en la línea axilar con electrodos de aguja subdérmica. Los niveles $T_{7}-T_{12}$ se colocan en la musculatura abdominal, en la línea mamaria, situados en la mitad imaginaria entre el límite inferior de la décima costilla y la cresta iliaca, con electrodos de aguja subdérmica, en una posición lineal entre la mama y la cresta iliaca antero superior. Se considera positivo si se evoca respuesta muscular con intensidades de estimulación inferiores a $6 \mathrm{~mA}$.

\section{REFERENCIAS}

1. Samdani AF et al. Triggered electromyography for placement of thoracic pedicle screws: is it reliable? Eur Spine J (2011) 20:869-74.

2. Rodriguez-Olaverri JC et al. Using triggered electromyographic threshold in the intercosta muscles to evaluate the accuracy of upper thoracic pedicle screw placement (T3-T6). Spine 2008;33, Number 7,194-7.

3. Suk SI et al. Thoracic pedicle screw fixation in spinal deformities: are they really safe? Spine 2001:26:2049-57.

4. Donahue ML et al. Pulse train stimulation for detecting medial malpositioning of thoracic pedicle screws. Spine 2008;33, Number 12, 378-85.

5. Shi YB, Binette M, Martin WH, et al. Electrical stimulation for intraoperative evaluation of thoracic pedicle screw placement. Spine 2003;28:595-601.

6. Lewis SJ, Lenke LG, Raynor B, et al. Triggered electromyographic threshold for accuracy of thoracic pedicle screw placement in a porcine model. Spine 2001:26:2384-9.

7. Owen $\mathrm{JH}$ et al. The use of mechanically elicited electromyograms to protect nerve roots during surgery for spinal degeneration. Spine 1994;19:1704-10.

8. Raynor BL, Lenke LG, Kim Y et al. Can triggered electromyograph trhesholds predict safe

\section{RESULTADOS}

Se implantaron un total de 421 tornillos torácicos, con la siguiente distribución: 29 en cirugía de escoliosis (63.04\%); 5 en cirugía de cifosis (10.86\%); 8 en fracturas del segmento torácico $(17.39 \%)$ y 4 en Cirugía reconstructiva (8.69\%). En todos los pacientes realizamos artrodesis posterolateral. Tuvimos alertas a la estimulación en 25 tornillos (5.93\%). A todos los pacientes se les realizó una radiografía de control postoperatoria, resultando 22 tornillos $(5.2 \%)$ medializados. Realizamos TAC en 17 pacientes (37\%), con ningún tornillo en posición I y 10 tornillos en posición II (8.5\%).

\section{DISCUSIÓN}

En nuestra serie, tuvimos 25 tornillos con alertas en la cirugía (5.93\% del total de tornillos torácicos registrados). El criterio electromiográfico elegido fue de valores inferiores a $6 \mathrm{~mA}^{7}$. En las radiografías hechas en el postoperatorio, se consideraron 22 tornillos como medializados $(5,2 \%)$ siguiendo los criterios descritos por Raynor y cols. ${ }^{8}$. En el escáner de control (TAC), no encontramos ningún tornillo en la categoría I (tornillos con una o más roscas mediales, intracanal), y 10 (8.5\%) en categoría $I^{4}$, recordemos que la categoría II se refiere a tornillos que tienen una o más roscas muy próximas a la pared medial del pedículo y que podrían haberla roto, aunque son considerados aceptables desde el punto de vista clínico.

A pesar de que la electromiografía (EMG) con tren de impulsos es una técnica aceptada para la correcta monitorización de la instrumentación transpedicular en la columna lumbar ${ }^{10}$, sigue existiendo controversia sobre su fiabilidad a nivel del segmento torácico ${ }^{1}$, si bien otros autores, como Rodríguez-Olaverri' ${ }^{2}$, han demostrado su utilidad en la instrumentación de niveles torácicos altos.

Por otro lado, la utilización de electrodos en los músculos intercostales en la línea axilar media ha mejorado la predicción de la posición de los tornillos ${ }^{4}$, existiendo también estudios experimentales sobre el uso de la EMG, consiguiendo evocar respuestas de los músculos intercostales contralaterales ${ }^{11}$

El uso de la EMG comparándolo con el TAC postoperatorio, muestra que valores $>6 \mathrm{~mA}$ tienen muchas posibilidades de caer en zona segura $^{12}$. La estimulación de los tornillos en el pedículo es una ayuda importante, junto con la palpación manual, para detectar roturas de la pared pedicular, incluso en niveles torácicos altos ${ }^{13}$. La EMG es un acertado predictor de la posición del tornillo en la columna torácica, con una sensibilidad del $94 \%$ y una especificidad del $90 \%$. Es muy importante que el miotomo de la raíz nerviosa testada ,esté monitorizada ${ }^{14}$.

\section{CONCLUSIONES}

En base a nuestros resultados, podemos enumerar las siguientes conclusiones:

El uso de tornillos pediculares torácicos es una técnica exigente, con una curva de aprendizaje larga no exenta de riesgos.

Los estudios de conducción nerviosa intraoperatorios (EMG) constituyen un método fiable en la detección de tornillos torácicos con malposición medial.

El uso de la EMG es aplicable no sólo a segmentos torácicos bajos, sino también a los segmentos altos.

Por último, esta técnica nos ha permitido reducir considerablemente la escopia intraoperatoria. thoracic pedicle screw placement? Spine 2001;27;2030-5.

9. Kim YJ, Lenke LG, Cheh G, Riew KD. Evaluation of pedicle screw placement in the deformed spine using intraoperative plain radiograph: a comparison with computerized tomography. Spine 2005:30:2084-8.

10. Raynor BL, Lenke LG, Bridwell KH, Taylor BA, Padberg AM. Correlation between low trig gered electromyographic thresholds and lumbar pedicle screw malposition. Analysis of 4587 screws. Spine 2007:32:2673-8.

11. De Blas G, Burgos J, Regidor I, Barrios C, Solá R, García-Urquiza S, Hevia E. Recording diffusion responses from contralateral intercostal muscles after stimulus triggered electromyography .Spine 2009;34;11:E391-6

12. Duffy MF, Phillips JH, Knapp D, Herrera Soto JA. Usefulness of electromyography compared to computed tomography scans in pedicle screw placement. Spine 2010;35;2:ppE43-8.

13. Norton JA, Hedden DM. Monitoring placement of high thoracic pedicle screws by triggered electromyography of the intercostal muscles. Can J Surg 2009;52;3:E47-8.

14. Danesh-Clough T, Taylor P, Hodgson B, Walton M. The use of evoked EMG in detecting misplaced thoracolumbar pedicle screws. Spine 2001:26:12:1313-6. 